\title{
Occupational noise exposure assessment using $0 *$ NET and its application to a study of hearing loss in the US general population
}

\author{
Yoon-Hyeong Choi, ${ }^{1}$ Howard Hu, ${ }^{1,2}$ SangWoo Tak, ${ }^{3}$ Bhramar Mukherjee, ${ }^{4}$ \\ Sung Kyun Park ${ }^{1,2}$
}

\begin{abstract}
- Additional materials are published online only. To view these files please visit the journal online (http://oem.bmj. com/content/69/3.toc)
\end{abstract}

1 Department of Environmental Health Sciences, University of Michigan School of Public Health, Ann Arbor, Michigan, USA

${ }^{2}$ Department of Epidemiology, University of Michigan School of Public Health, Ann Arbor,

Michigan, USA

${ }^{3}$ Division of Surveillance, Hazard Evaluation, and Field Studies, National Institute for Occupational Safety and Health, Cincinnati, Ohio, USA

${ }^{4}$ Department of Biostatistics, University of Michigan School of Public Health, Ann Arbor. Michigan, USA

\section{Correspondence to} Sung Kyun Park, M5541 SPH II, 1415 Washington Heights, Ann Arbor, Ml 48109-2029, USA sungkyun@umich.edu

Accepted 8 June 2011 Published Online First 1 July 2011

\begin{abstract}
Objectives Although occupational noise is a well known risk factor for hearing loss, little epidemiological evidence has been reported on its association with hearing loss in the general population, in part, because of the difficulty in exposure assessment. This study introduced a quantitative occupational noise exposure assessment tool using the Occupational Information Network ( $\left.0^{*} \mathrm{NET}\right)$ database and evaluated its applicability for epidemiological research using data from the National Health and Nutrition Examination Survey (NHANES) 1999-2004.
\end{abstract}

Methods The 0*NET noise exposure data were assessed by questionnaires across numerous occupations, asking the frequency of exposure to sounds and noise levels that are distracting and uncomfortable (with five possible responses from 'never' to 'every day'). Means of the O*NET noise scores were computed to correspond to NHANES occupational categories and assigned to 3828 adults aged 20-69 years, who participated in the 1999-2004 NHANES. Pure-tone averages (PTA) of hearing thresholds at 0.5, 1, 2 and $4 \mathrm{kHz}$ were computed, and hearing loss was defined as a PTA $>25 \mathrm{~dB}$ in either ear. Linear and logistic regression models with either continuous or quintiles of the $0^{*}$ NET noise scores were fitted on log-transformed PTA and binary hearing loss, respectively.

Results Noise scores ranged from 1.80 to 4.37 with mean $\pm S E$ of $3.06 \pm 0.02$. After controlling for potential confounders, the highest (vs lowest) noise score quintile had a $22.5 \%(95 \% \mathrm{Cl} 11.0 \%$ to $35.2 \%)$ increase in PTA, and there was a linear dose-dependent trend across the quintiles of noise scores ( $p$ trend $<0.0001$ ). The adjusted $\mathrm{OR}$ for hearing loss comparing the highest with the lowest noise score quintiles was 2.1 (95\% $\mathrm{Cl} 1.2$ to 3.6$)$. Conclusion This study suggests that the $0^{*}$ NET noise score is a useful tool for examining occupational noiseinduced health effects in the general population in the absence of actual occupational noise exposure assessment data.

\section{INTRODUCTION}

Hearing loss is one of the leading chronic health disabilities experienced by older adults. ${ }^{1}$ More than 35 million Americans aged 18 years and older suffer from hearing loss, and the number of people with hearing loss tends to increase dramatically with advancing age. ${ }^{2}$ In addition to ageing, noise is one of the most important determinants of hearing

\section{What this paper adds}

- This study introduced a new exposure assessment tool for occupational noise using the Occupational Information Network (0*NET) database and found a strong association between occupational noise estimated using this new assessment tool and hearing impairment in the US general population.

- Our results extend evidence of noise-induced hearing loss, studied almost exclusively among workers exposed to extremely high noise, to a sample of the general population with a variety of different noise exposures and reinforce occupational noise as an important risk factor for hearing loss.

loss. While hearing loss could occur purely by noise induction, it usually occurs from a combination of different factors.

Occupational noise exposure has been associated with hearing loss, especially among workers with high noise exposure levels. ${ }^{3-7}$ Approximately 16\% of hearing impairment worldwide is attributed to occupational noise. $^{7-9}$ In the USA, about 5-30 million workers are exposed to noise levels at work that put them at risk of hearing loss. ${ }^{10}$

Hearing loss can be diagnosed through review of an audiogram, regardless of whether the hearing loss is caused by noise or other factors, such as ageing. ${ }^{11} 12$ Noise-induced hearing loss usually begins at 3, 4 or $6 \mathrm{kHz}$ (higher frequencies). With noise-induced hearing loss, thresholds at these frequencies are higher (indicating hearing loss) than at that of 0.5 and $1 \mathrm{kHz}$ (lower frequencies) and at $8 \mathrm{kHz}$ (recovery), which is named a noise notch. ${ }^{12} 13$ In contrast, the audiogram of age-related hearing loss shows substantial down-sloping (higher thresholds) in higher frequencies. ${ }^{11} 13$ The association between noise exposure and noise notch allows us to observe the distinct effect of noise exposure on noise-induced hearing loss while excluding the effect of changes in hearing ability by other factors.

Although previous epidemiological studies have consistently shown a positive association between occupational noise exposure and hearing loss, few studies have been able to measure cumulative personal noise exposure in the general population. 
Occupation and/or industry classifications can be used through job-exposure matrix (JEM), ${ }^{14} 15$ and several studies conducted in the USA and Europe reported an association between JEMestimated noise exposure and hearing loss. ${ }^{6} 816$ However, such JEMs are limited to occupations and industries with high noise exposures, which preclude studies of the health effect of occupational noise exposure in the general population.

Recent studies have reported that the Occupational Information Network ( $\mathrm{O}^{*} \mathrm{NET}$ ) has the potential to serve as JEM for selected environmental factors, applying it to national health surveys to examine associations with health outcomes. ${ }^{17} 18$ This study used the O*NET database as a surrogate measure for occupational noise exposure estimates. The O*NET is the survey-based database system generated by the US Department of Labor providing information on skills, abilities, knowledge, work activities and interests across occupational groups. ${ }^{19}$ Data were collected through questionnaires from employees selected in every occupation group coded as the Standardized Occupation Codes (SOC) and were scored by mean scales in each SOC group. Because a question about occupational noise exposure is included in the questionnaire, mean scores of such a question may provide quantitative measures of occupational noise exposure in all occupation groups, those occupations with high noise exposure as well as those with low exposure. This allows us to evaluate the potential health effects of noise within various occupational groups of the general population. An important limitation of this approach is the fact that it cannot be validated as a method of assessing occupational exposure without being compared to direct measurements of workplace noise in each occupation. Nonetheless, if the $\mathrm{O}^{*} \mathrm{NET}$ noise estimates have a significant association with hearing loss in a well-defined population with a wide range of occupation groups, the applicability of this method may be confirmed.

The aims of this study were to introduce a quantitative occupational noise exposure assessment tool using $\mathrm{O}^{*} \mathrm{NET}$ and to evaluate its applicability using data from the National Health and Nutrition Examination Survey (NHANES) 1999-2004 in the general US population.

\section{MATERIALS AND METHODS \\ 0 *NET noise exposure assessment}

The O*NET is a publicly available on-line database that describes occupational features across SOC taxonomy. This study used the recent version 'O*NET 12.0' available at the O*NET website (http://www.onetcenter.org/database.html\#download) or the National Crosswalk Service Center (http://www.xwalkcenter. org). We extracted the data of the occupational noise scale scores as the element name 'Sounds, Noise Levels are Distracting, etc' (element ID IV.C.2.b.1.a) across 801 SOC groups. Occupational noise exposure was scored by frequency-type answers to the question, 'In your current job, how often are you exposed to sounds and noise levels that are distracting and uncomfortable?'. ${ }^{20}$ Five responses are possible, 'never (1)', 'once a year or more but not every month (2)', 'once a month or more but not every week (3)', 'once a week or more but not every day (4)' and 'every day (5)'. ${ }^{21}$ An example of the O*NET database structure (O*NET SOC code, noise estimates, SE and survey sample size) is presented in supplemental table 1 . We used the mean of responses in each 801 SOC occupation as a proxy measure of occupational noise exposure. For example, the category 'accountants' (SOC: 13-2011.01) has the mean score of 1.49 $(\mathrm{SE}=0.24)$, whereas the category 'construction carpenters' (SOC: 47-2031.01) has $4.17(\mathrm{SE}=0.26)$. If the score is closer to 5, most workers in that job category are exposed daily to 'distracting and uncomfortable noise levels'. Likewise, if the score is close to 1, workers in that category are almost never exposed to such noise levels. The mean $\mathrm{O}^{*} \mathrm{NET}$ noise scores, therefore, represent the probability of a worker in a certain job category being exposed to 'distracting and uncomfortable sounds and noise' and do not reflect the loudness of sound wave (such as decibels).

\section{Application of the $0 *$ NET noise estimates to a hearing loss study} Study population

The NHANES, conducted by the National Center for Health Statistics (NCHS) since the early 1960s, is an ongoing series of cross-sectional surveys designed to assess health and nutritional status in the civilian non-institutionalised US population.

In NHANES 1999-2004, half of the subjects aged 20-69 years were randomly selected to participate in the Audiometry Examination Component. Subjects were excluded if they used hearing aids that were not able to be removed for testing or had sufficient ear pain at the time of the examination that they could not tolerate headphones. ${ }^{22}$ The eligible sample size was 5742 participants: 1807 in 1999-2000, 2046 in 2001-2002 and 1889 in 2003-2004

\section{Audiometric measures}

Audiometry examination was performed in a sound-isolated room in the mobile examination centre by health technicians trained by a National Institute for Occupational Safety and Health-certified audiologist. Instrumentation for the Audiometry Component included an Interacoustics Model AD226 audiometer with standard TDH-39 headphones and EtymoticEar Tone $3 \mathrm{~A}$ insert earphones. ${ }^{22}$ Pure-tone air conduction hearing thresholds were obtained on both ears at frequencies of $0.5,1,2,3,4,6$ and $8 \mathrm{kHz}$ across an intensity range of -10 to $120 \mathrm{~dB}$. Participants with missing value at one or more frequencies were excluded from analysis. Procedural details in collecting the audiometric data have been described elsewhere. ${ }^{22} 23$

We computed hearing thresholds (decibels) at speech frequencies as a pure-tone average (PTA) of 0.5, 1, 2 and $4 \mathrm{kHz}$ and the hearing thresholds at high frequencies as a pure-tone average (high PTA) of 3,4 and $6 \mathrm{kHz} .{ }^{24}$ According to classification by the WHO, hearing loss is defined as PTA greater than $25 \mathrm{~dB}$ in either ear. ${ }^{24} 25$ Noise notch was defined as the hearing threshold at 3, 4 and/or $6 \mathrm{kHz}$ at least $10 \mathrm{~dB}$ greater than at 1 or $2 \mathrm{kHz}$ and at least $10 \mathrm{~dB}$ greater than at 6 or $8 \mathrm{kHz}{ }^{11} 12$

Of the initial sample of 5742 participants eligible for inclusion in the audiometry examination, 324 (6\%) were excluded from the analysis because a test was not performed at all and 152 $(3 \%)$ were excluded because tests have missing values at one or more frequencies. As an additional measure of reliability of participant responses, all audiograms tested the $1 \mathrm{kHz}$ frequency twice in each ear. ${ }^{22}$ Three participants were excluded because there was $>10 \mathrm{~dB}$ difference between the $1 \mathrm{kHz}$ test-retest thresholds. $^{24}$ An additional 452 participants were excluded because of unilateral hearing loss defined as $>10 \mathrm{~dB}$ difference between the PTAs of left and right ears. Therefore, audiometric results for 4811 participants were included for analysis in the present study.

\section{Occupational noise estimates}

Participant occupation information was obtained from the occupation questionnaire, which contains personal interview data on employment and variables relating to the work environment, such as workplace noise exposure history. ${ }^{26}$ We used the participant's longest job as a measure of past noise exposure. The longest job information was obtained by asking the 
question directly: "What kind of work were you doing the longest?'. ${ }^{27}$ Occupation data were then coded by trained coders using the US Census Bureau's Census 1990 or 2000 indexes of occupation, which are the three-digit NCHS Occupational Classification Source Codes. ${ }^{28}$ In the publicly available NHANES data, these codes were collapsed into 41 occupation categories. ${ }^{27}$ Of the 4811 participants with available audiometric measurements, 4547 had information available on their longest job-2498 participants who answered their longest job plus an additional 2049 participants who answered their longest job were the same as their current job and for whom the current job code was assigned as their longest job. To link the O*NET noise estimates to the 41 occupation categories, we grouped the 801 O*NET SOCs available into the corresponding NHANES occupation categories and computed the averages of the $\mathrm{O}^{*} \mathrm{NET}$ noise scores in each category. Because military occupations were not included in the $O *$ NET survey, we could not generate an $O * N E T$ noise score for the military occupation group and so resulted in 40 occupation groups for analysis. After linking O*NET noise estimates to occupation categories, we assigned an average noise score to each participant. Of the 4547 participants with available longest job information, we excluded 66 participants whose longest jobs were not coded to any of the 41 occupation groups and 59 participants who had military occupation, yielding 4422 participants available for statistical analyses.

We also computed the weighted averages of O*NET noise scores for the 40 NHANES occupation groups, accounting for the SEs of the noise scores in each $\mathrm{O}^{*} \mathrm{NET}$ SOC group and which reflect the precision of the $O *$ NET survey data (see supplemental table 1). Because SEs were not available for 58 of the 801 SOCs, we used the unweighted averages as our primary index of occupational noise and examined the weighted ones as a sensitivity analysis. The unweighted and weighted average $O$ *NET scores for 40 occupation categories are presented in table 1.

Table 1 Unweighted and weighted averages of $0 *$ NET occupational noise scores by 40 NHANES occupation groups

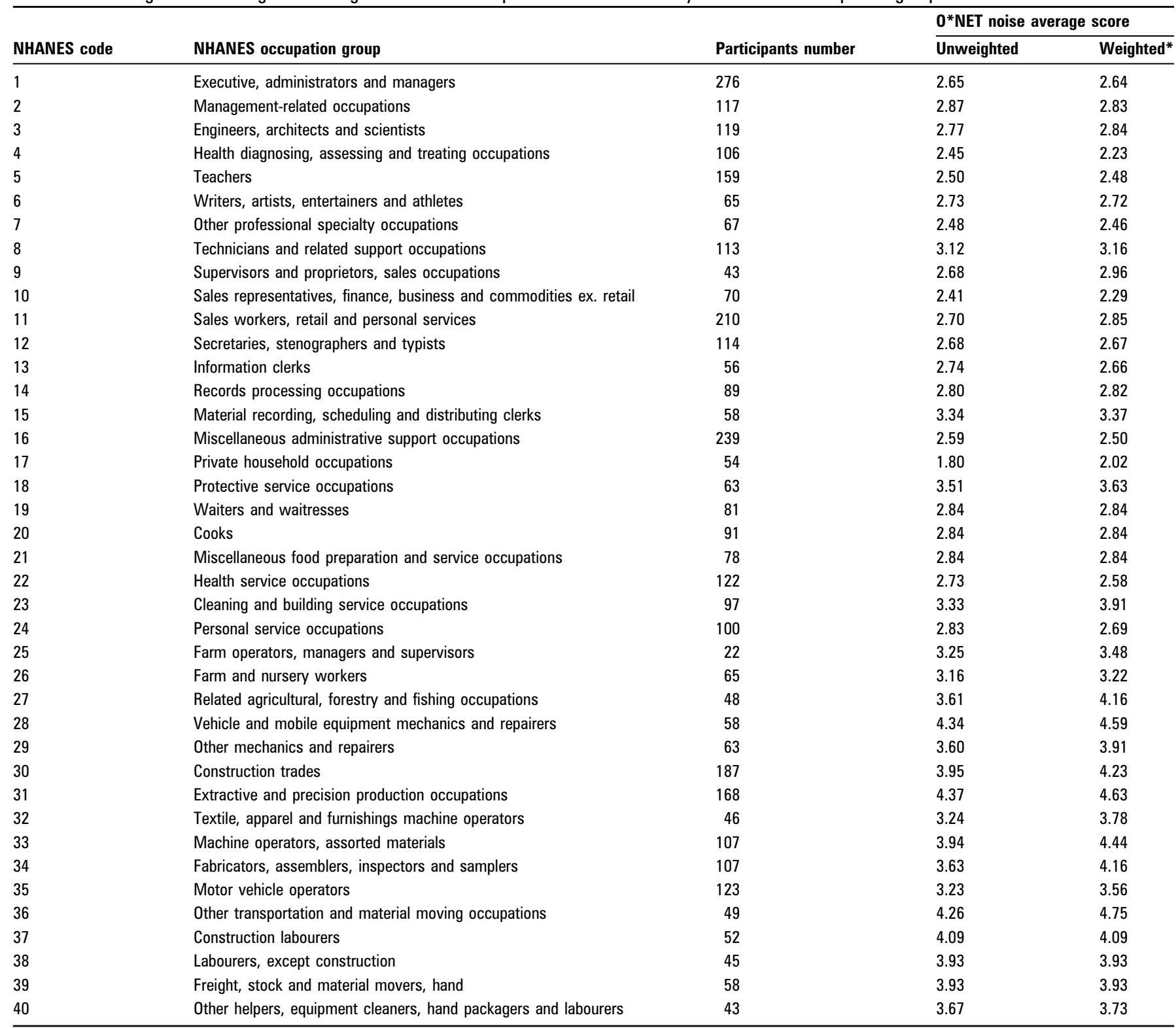

${ }^{*}$ Weighted average was defined $\sum_{i=i}^{p} \frac{\left(0^{*} \text { NETscore }_{i}\right) / S E_{i}}{\sum_{i=1}^{p}\left(1 / S E_{i}\right)}$ as where $i$ is an individual job title in $0 *$ NET Standard 0 ccupation Classification (SOC) and $\mathrm{p}$ is the total number of $0 *$ NET SOCs 
The characteristics between participants with and without the longest job information were similar in terms of prevalence of hearing loss, age, body mass index (BMI) and the status of hypertension and diabetes. Compared to included participants, excluded participants were less likely to be male, nonHispanic white and smokers, less educated, and less exposed to occupational noise (see supplemental table 2).

\section{Demographic and hearing-related variables}

Other demographic and hearing-related variables were obtained from the NHANES questionnaires. BMI was calculated as weight in kilograms/height in metres squared (missing for 49 participants). Use of ototoxic medication was counted when participants reported medications of aminoglycoside, loop diuretics, antineoplastic drugs or non-steroidal anti-inflammatory drugs (missing for five participants). Smoking pack-years were computed and grouped into non-smokers, smokers who smoked $<20$ pack-years or smokers who smoked $>20$ pack-years (missing for 392 participants). Hypertension was defined as selfreported physician diagnosis, use of antihypertensive medication, systolic blood pressure $\geq 140 \mathrm{~mm} \mathrm{Hg}$ or diastolic blood pressure $\geq 90 \mathrm{~mm} \mathrm{Hg}$ at the time of examination (missing for 189 participants). Diabetes mellitus was defined as those who self-reported either previous physician diagnosis or use of antihyperglycemic medication (missing for two participants). Nonoccupational noise exposures were determined by audiometry questionnaires asking if the subject had ever been exposed outside of work to the noise of a firearm for a mean of at least once a month for 1 year (missing for five participants) and if the subject had ever been exposed outside of work to loud noise (eg, power tools or loud music) for a mean of at least once a month for 1 year (missing for six participants). Socioeconomic status, such as education attainment, may be a potential confounder, but we did not consider it because education is highly correlated with job title used for our O*NET noise estimates, and controlling for such a socioeconomic status may result in over adjustment for our exposure effect.

Our study sample was limited to adults who had complete information on these important covariates, and therefore, a total of 3828 participants were available for data analyses.

\section{Statistical analysis}

All statistical analyses were performed using SAS survey procedures (SAS V.9.2) and the Rsurvey package (R V.2.9.1) to account for the complex survey design of the NHANES. ${ }^{1} 29$ Sample weights for the combined 6-year sample were used as per NCHS recommendations in order to provide annual national estimates, which accounted for the unequal probabilities of selection due to over sampling and non-response. ${ }^{24}$

Linear regressions were used for continuous hearing thresholds in each frequency and PTA. Hearing threshold outcomes were log-transformed to normalise distributions. Eighty subjects $(2.1 \%)$ with better-than-normal hearing had zero or negative hearing thresholds. We excluded these subjects to better interpret regression results of log-transformed thresholds in our primary linear regression analyses. Linear regressions including all available subjects were considered in sensitivity analyses to evaluate the effect of the exclusion. In sensitivity analyses, a constant 6 was added before log-transformation to make all hearing threshold values positive and the resulting data as normal as possible. We examined the O*NET noise score as a continuous variable and in quintiles. For the latter, we tested for linear trend across quintiles using ordinal terms. For dichotomous hearing loss and noise notch outcomes, we determined the OR and 95\% CI using logistic regression models. In multiple regression analyses, we identified a priori those covariates that needed to be controlled for, based on biological consideration and the current state of the literature: age (years), sex, race/ethnicity, BMI (kilograms per square metre), cigarette smoking (pack-years), ototoxic medication, hypertension, diabetes mellitus, firearm noise exposure and recreation noise exposure. ${ }^{30} \mathrm{We}$ fit age and age-squared to capture non-linear effects of age. To identify influence of potential confounders, we developed sequential models: (1) age, sex and race/ethnicity adjusted; (2) additionally adjusted for BMI, ototoxic medication, cigarette smoking, current diagnosis of hypertension and current diagnosis of diabetes and (3) additionally adjusted for recreational noise and firearm noise exposures. We examined subgroup analyses by age, sex and race/ethnicity to identify the most susceptible subpopulations.

\section{RESULTS \\ 0*NET noise exposure assessment}

Table 1 shows the unweighted and weighted averages of $O^{*}$ NET noise scores by 40 occupation groups. 'Private household occupations' had the lowest noise score in both unweighted (1.80) and weighted averages (2.02), whereas 'extractive and precision production occupations' and 'other transportation and material moving occupations' were highest in unweighted (4.37) and weighted (4.63) averages, respectively. We used the unweighted scores in all subsequent analyses.

\section{Application to hearing loss study General characteristics}

Table 2 shows descriptive characteristics of study participants. Overall, 456 subjects $(11.9 \%)$ had a mild or greater hearing loss. After accounting for sampling weights, cluster and strata of the NHANES complex design, the mean of O*NET noise scores in the entire population was $3.06(\mathrm{SE}=0.02)$, and subjects with hearing loss had a significantly higher noise score than those without hearing loss (3.26 ( $\mathrm{SE}=0.04)$ vs $3.04(\mathrm{SE}=0.02)$ ). The means of $\mathrm{O}^{*} \mathrm{NET}$ scores in the entire population and for subjects with and without hearing loss which were not considered sampling weights were $3.09(\mathrm{SD}=0.60), 3.26(\mathrm{SD}=0.65)$ and 3.07 $(\mathrm{SD}=0.59)$, respectively. Distributions of the $\mathrm{O}$ NET noise scores in the NHANES participants are shown in supplemental figure 1. Subjects with hearing loss were older (54.8 vs 40.4 years), more likely to be male ( $66 \%$ vs $46 \%$ ), non-Hispanic white ( $80 \%$ vs $71 \%$ ) and ever smoker (59\% vs $46 \%$ ), used ototoxic medication ( $24 \%$ vs $15 \%$ ), less educated (percentage of greater than high school diploma $43 \%$ vs $60 \%$ ), more likely to be exposed to occupational noise (45\% vs $32 \%$ ) and firearm noise ( $13 \%$ vs $7 \%$ ), and more likely to have hypertension ( $43 \%$ vs $21 \%$ ) and diabetes (12\% vs $3 \%$ ) than those without hearing loss.

Table 3 presents the distributions of participant characteristics by quintiles of the O*NET noise scores. Subjects with higher occupational noise were more likely to be male and of race/ ethnicity other than non-Hispanic white, more likely to have higher hearing thresholds, less educated and more likely to be exposed to firearm and recreational noises than those with lower occupational noise.

\section{Occupational noise exposure and hearing thresholds}

Table 4 shows per cent changes in PTA in associations with the $\mathrm{O}$ *NET score as a continuous variable and in quintiles in various covariate-adjusted models. An increase in occupational noise exposure either as a continuous variable or in quintiles was significantly associated with higher (poorer) hearing thresholds. In the fully adjusted model (model $\mathrm{C}$ ), subjects in the highest 
Table 2 Participant characteristics by hearing loss status $\left(n=3828^{*}\right)$

\begin{tabular}{|c|c|c|c|c|}
\hline Characteristic & All participants $(n=3828)$ & Not hearing loss $(n=3372)$ & Hearing loss $\dagger(n=456)$ & p Value $\neq$ \\
\hline 0*NET noise§ & $3.06( \pm 0.02)$ & $3.04( \pm 0.02)$ & $3.26( \pm 0.04)$ & $<0.0001$ \\
\hline 0*NET noise§ (weighted) & $3.15( \pm 0.02)$ & $3.12( \pm 0.02)$ & $3.39( \pm 0.05)$ & $<0.0001$ \\
\hline Age (y) & $41.96( \pm 0.27)$ & $40.35( \pm 0.27)$ & $54.81( \pm 0.67)$ & $<0.0001$ \\
\hline Pure-tone average hearing thresholds $\Phi(\mathrm{dB})$ & $12.72( \pm 0.24)$ & $10.96( \pm 0.16)$ & $27.00( \pm 0.70)$ & $<0.0001$ \\
\hline Noise notch ${ }^{* *}(\%)$ & 17.6 & 16.8 & 23.7 & $<0.0001$ \\
\hline Sex (male \%) & 48.5 & 46.3 & 66.2 & $<0.0001$ \\
\hline Non-Hispanic black & 10.7 & 11.5 & 4.9 & \\
\hline Mexican American & 6.7 & 7.1 & 3.6 & \\
\hline Other & 10.3 & 10.2 & 11.3 & \\
\hline Ototoxic medication (current use \%) & 15.9 & 14.8 & 24.4 & 0.0013 \\
\hline Cumulative cigarette pack-years (\%) & & & & $<0.0001$ \\
\hline Diabetes mellitus (\%) & 4.3 & 3.4 & 11.5 & $<0.0001$ \\
\hline Noise exposure at firearm (exposed \%) & 7.4 & 6.6 & 13.2 & 0.0010 \\
\hline Noise exposure at recreation (exposed \%) & 25.9 & 25.4 & 29.6 & 0.1341 \\
\hline Noise exposure at job†† (exposed \%) & 33.0 & 31.5 & 45.4 & $<0.0001$ \\
\hline
\end{tabular}

*Participants $(n=3828)$ are the individuals having all interest variables in this study: hearing thresholds, hearing loss, noise, age, body mass index, sex, race/ethnicity, ototoxic medication, cumulative cigarette pack-years, hypertension, diabetes mellitus, firearm noise exposure and recreation noise exposure.

† Hearing loss was defined as pure-tone average at speech frequencies $>25 \mathrm{~dB}$.

$\neq$ Survey $t$ test (age adjusted) for continuous variables and survey (Rao-Scott) $\chi^{2}$ test for categorical variables were used.

$\S 0 *$ NET noise score $(1<$ Noise scale $<5)$.

TPure-tone average at speech frequencies at $0.5,1,2$ and $4 \mathrm{kHz}$.

**Noise notch (hearing threshold at $3,4 \mathrm{and} / \mathrm{or} 6 \mathrm{kHz}$ is at least $10 \mathrm{~dB}$ greater than at 1 or $2 \mathrm{kHz}$ and at least $10 \mathrm{~dB}$ greater than at 6 or $8 \mathrm{kHz}$ ).

††Noise exposure at job was defined as ever exposure to loud noise at work for at least 3 months.

$0 *$ NET, Occupational Information Network.

noise quintile had $22.5 \%$ (95\% CI $11.0 \%$ to $35.2 \%$ ) higher hearing thresholds than those in the lowest quintile. A unit increase in the O*NET noise score was associated with a $15.4 \%$ $(95 \%$ CI $9.7 \%$ to $21.5 \%)$ increase in hearing thresholds in the fully adjusted model. The same trends were also observed in high PTA (see supplemental table 3 ) and all individual frequencies (see supplemental figure 2). Table 4 was designed to show results from the linear regression of log-transformed PTA with the O*NET score, which excludes the subjects with zero and negative hearing thresholds (2.09\%) for better interpretation of log-transformation. Supplemental table 4, panel A versus B., compares results in subjects with only positive hearing thresholds with results in all available subjects. Panel A was designed to show results from the linear regression of log-transformed (PTA $+6)$ with the O*NET score in all available subjects, whereas panel $\mathrm{B}$ was designed to show results from the linear regression of log-transformed (PTA+6) with the O*NET score in subjects with only positive hearing thresholds, the same subjects as

Table 3 Characteristics of study population by noise exposure quintile at longest job

\begin{tabular}{|c|c|c|c|c|c|c|}
\hline & \multicolumn{5}{|c|}{ 0*NET noise exposure scores at longest job $(n=3828)$} & \multirow[b]{2}{*}{$\mathrm{p}$ Trend } \\
\hline & $\begin{array}{l}\text { Quintile 1 } \\
(1.795-2.588) \\
(\mathrm{n}=695)\end{array}$ & $\begin{array}{l}\text { Ouintile } 2 \\
(2.653-2.729) \\
(n=830)\end{array}$ & $\begin{array}{l}\text { Quintile 3 } \\
(2.737-2.868) \\
(\mathrm{n}=731)\end{array}$ & $\begin{array}{l}\text { Ouintile } 4 \\
(3.121-3.631) \\
(n=805)\end{array}$ & $\begin{array}{l}\text { Quintile } 5 \\
(3.667-4.368) \\
(\mathrm{n}=767)\end{array}$ & \\
\hline Age (y) & $43.8( \pm 0.5)$ & $41.8( \pm 0.5)$ & $40.9( \pm 0.5)$ & $42.0( \pm 0.6)$ & $41.4( \pm 0.6)$ & 0.0066 \\
\hline Hearing loss $†(\%)$ & 8.8 & 8.7 & 8.5 & 12.8 & 17.8 & $<0.0001$ \\
\hline Noise notch $\neq(\%)$ & 13.8 & 14.2 & 11.8 & 22.4 & 27.0 & $<0.0001$ \\
\hline Non-Hispanic white & 76.25 & 75.40 & 75.16 & 64.82 & 68.63 & \\
\hline Non-Hispanic black & 10.09 & 10.30 & 9.70 & 13.81 & 10.12 & \\
\hline Mexican American & 4.02 & 4.66 & 4.73 & 10.69 & 9.98 & \\
\hline Other & 9.64 & 9.63 & 10.41 & 10.68 & 11.27 & \\
\hline Noise exposure at firearm (exposed \%) & 3.0 & 7.0 & 4.3 & 10.2 & 12.8 & $<0.0001$ \\
\hline Noise exposure at recreation (exposed \%) & 18.9 & 24.2 & 21.6 & 28.9 & 36.6 & $<0.0001$ \\
\hline
\end{tabular}

*PTA (pure-tone average) at speech frequencies at $0.5,1,2$ and $4 \mathrm{kHz}$, age adjusted.

tHearing loss (PTA at speech frequencies $>25 \mathrm{~dB}$ ).

$\ddagger$ Noise notch (hearing threshold at $3,4 \mathrm{and} / \mathrm{or} 6 \mathrm{kHz}$ is at least $10 \mathrm{~dB}$ greater than at 1 or $2 \mathrm{kHz}$ and at least $10 \mathrm{~dB}$ greater than at 6 or $8 \mathrm{kHz}$ ).

$0 *$ NET, Occupational Information Network. 
Table 4 Per cent change (95\% Cls) of hearing thresholds (decibels) by noise exposure levels at longest job

\begin{tabular}{|c|c|c|c|c|}
\hline Variables & No. & Model A* & Model B十 & Model C $\neq$ \\
\hline $0 *$ NET Noise (unit score) $\S$ & & 18.41 (12.23 to 24.93$)$ & 16.01 (10.09 to 22.25$)$ & 15.43 (9.70 to 21.45$)$ \\
\hline \multicolumn{5}{|l|}{$0 * N E T$ noise quintile } \\
\hline Quintile $1(1.795-2.588)$ & 680 & 0 (Reference) & 0 (Reference) & 0 (Reference) \\
\hline Quintile $2(2.653-2.729)$ & 807 & $2.90(-5.61$ to 12.17$)$ & $1.89(-6.30$ to 10.78$)$ & $1.44(-6.71$ to 10.31$)$ \\
\hline Quintile $3(2.737-2.868)$ & 711 & $0.72(-8.98$ to 11.45$)$ & $-0.81(-10.30$ to 9.68$)$ & $-0.90(-10.40$ to 9.61$)$ \\
\hline Quintile 4 (3.121-3.631) & 793 & $17.24(6.20$ to 29.42$)$ & 14.02 (3.32 to 25.82$)$ & 13.27 (2.87 to 24.72 ) \\
\hline Quintile 5 (3.667-4.368) & 757 & 27.97 (15.99 to 41.20$)$ & 23.66 (11.90 to 36.66$)$ & 22.48 (10.99 to 35.15$)$ \\
\hline $\mathrm{p}$ Trend & & $<0.0001$ & $<0.0001$ & $<0.0001$ \\
\hline
\end{tabular}

*Model $A$ was adjusted for age, age ${ }^{2}$, sex and race/ethnicity.

†Model B: model A+further adjusted for body mass index, ototoxic medication, cumulative cigarette pack-years, current diagnosis of hypertension and current diagnosis of diabetes.

$\neq$ Model C: model $B$ +further adjusted for recreation noise and firearm noise.

$\S$ Per cent change in hearing thresholds for one-unit score increase.

$0 *$ NET, Occupational Information Network.

table 4. From the comparison of supplemental table 4, panel A versus $B$, linear regression in subjects with only positive hearing thresholds showed consistent patterns to those of linear regression in all available subjects.

Occupational noise exposure and hearing loss and noise notch Table 5, panel A, shows the association between $\mathrm{O}^{*} \mathrm{NET}$ noise score and the risk of hearing loss in different covariate-adjusted models. There were significant dose-dependent relationships in all models, and further adjustment for potential confounders including ototoxic medication, cigarette smoking, hypertension, diabetes, recreation noise and firearm noise did not change the result. The fully adjusted OR for hearing loss comparing the highest versus the lowest quintiles was 2.07 (95\% CI 1.18 to 3.63$)$

Table 5, panel $\mathrm{B}$, shows OR for risk of the noise notch by quintiles of $\mathrm{O}^{*} \mathrm{NET}$ noise score. The association showed a statistically significant dose-dependent relationship, and the increase pattern in the risk of noise notch in association with O*NET noise score explains better the dose-dependent relationship between occupational noise and hearing loss than that in the OR of the risk of hearing loss by the O*NET noise score in panel A. The fully adjusted OR for noise notch comparing the highest versus the lowest quintiles was 1.51 (95\% CI 1.09 to 2.09).

As a sensitivity analysis, we also examined associations with the weighted O*NET noise scores. Overall, associations were similar to those with unweighted scores (supplemental tables 5 and 6).

\section{Effect of occupational noise at participants' subgroups}

We examined the association of the O*NET noise score, modelled as a continuous variable, with PTA in the subgroups by important demographic characteristics such as sex, age and race/ ethnicity. The association was strongest among the age group of 40-59 years, whereas it was less significant among the age group over 60 years. Men or non-Hispanic white also had higher O*NET noise-associated PTAs than did women or other race/ ethnic groups (see supplemental table 7).

\section{DISCUSSION}

The present study introduces a new occupational noise exposure assessment tool using the O*NET database, evaluating its applicability to an examination of noise-related adverse health

Table 5 ORs (95\% Cls) of hearing loss and noise notch by noise exposure levels at longest job

\begin{tabular}{|c|c|c|c|c|}
\hline Variables & & Model A* & Model B† & Model C $\neq$ \\
\hline A. ORs of hearing loss & Hearing loss no./participants no. & & & \\
\hline $0 *$ NET noise (unit score) $§$ & & $1.74(1.35$ to 2.26$)$ & $1.68(1.30$ to 2.18$)$ & $1.65(1.28$ to 2.13$)$ \\
\hline \multicolumn{5}{|l|}{$0 *$ NET noise quintile } \\
\hline Quintile 1 (1.795-2.588) & $65 / 695$ & 1 (Reference) & 1 (Reference) & 1 (Reference) \\
\hline Quintile 2 (2.653-2.729) & $76 / 830$ & $1.04(0.62$ to 1.72$)$ & 1.01 (0.60 to 1.69$)$ & $0.99(0.59$ to 1.65$)$ \\
\hline Quintile $3(2.737-2.868)$ & $67 / 731$ & $1.14(0.64$ to 2.03$)$ & $1.10(0.62$ to 1.96$)$ & $1.09(0.61$ to 1.95$)$ \\
\hline Quintile 4 (3.121-3.631) & $112 / 805$ & $1.61(0.96$ to 2.70$)$ & 1.50 (0.89 to 2.52$)$ & $1.43(0.87$ to 2.36$)$ \\
\hline Quintile 5 (3.667-4.368) & $136 / 767$ & $2.30(1.32$ to 4.01$)$ & $2.14(1.22$ to 3.75$)$ & 2.07 (1.18 to 3.63$)$ \\
\hline $\mathrm{p}$ Trend & & 0.001 & 0.0019 & 0.0026 \\
\hline B. ORs of noise notch & Noise notch no./participants no. & & & \\
\hline $0 *$ NET noise (unit score) $\S$ & & $1.45(1.20$ to 1.76$)$ & $1.43(1.18$ to 1.73$)$ & $1.41(1.17$ to 1.70$)$ \\
\hline \multicolumn{5}{|l|}{$0 * N E T$ noise quintile } \\
\hline Quintile 1 (1.795-2.588) & $101 / 695$ & 1 (Reference) & 1 (Reference) & 1 (Reference) \\
\hline Quintile 2 (2.653-2.729) & $119 / 830$ & $0.98(0.67$ to 1.42$)$ & 0.97 (0.67 to 1.42$)$ & $0.96(0.66$ to 1.40$)$ \\
\hline Quintile 3 (2.737-2.868) & 77/731 & $0.80(0.58$ to 1.11$)$ & $0.80(0.58$ to 1.10$)$ & $0.79(0.57$ to 1.09$)$ \\
\hline Quintile 4 (3.121-3.631) & $168 / 805$ & $1.40(1.05$ to 1.87$)$ & $1.37(1.02$ to 1.84$)$ & $1.35(1.00$ to 1.81$)$ \\
\hline Quintile 5 (3.667-4.368) & $190 / 767$ & 1.60 (1.16 to 2.20$)$ & 1.55 (1.12 to 2.14$)$ & 1.51 (1.09 to 2.09 ) \\
\hline $\mathrm{p}$ Trend & & 0.0016 & 0.0032 & 0.0045 \\
\hline
\end{tabular}

*Model $A$ was adjusted for age, age ${ }^{2}$, sex and race/ethnicity.

† Model B: model A+further adjusted for body mass index, ototoxic medication, cumulative cigarette pack-years, current diagnosis of hypertension and current diagnosis of diabetes.

¥Model $\mathrm{C}$ : model $\mathrm{B}$ +further adjusted for recreation noise and firearm noise.

$\S$ Per cent change in hearing thresholds for one-unit score increase.

0*NET, Occupational Information Network. 
effects in the general population using hearing loss, a wellestablished noise-induced health outcome.

Our findings suggest that the use of O*NET scores may provide enough variation in the proxy measure of occupational noise exposure so that it can be applied for the general population with a wide range of occupation groups. It should be noted that this study did not attempt to validate the O*NET scores as a surrogate for personal occupational noise exposure levels. Rather, we evaluated an applicability of the O*NET scores as a proxy measure in association with occupational noise-related health effects in the general population, given available job title information. We found a significant dose-response relationship of O*NET noise scores with hearing loss and noise notch in NHANES, confirming that O*NET scores would be useful for examining noise-related health effects in the absence of personal occupational noise exposure data. Our results also extend evidence of noise-induced hearing loss in workers with extremely high noise exposure to the general population with low noise exposure, reinforcing occupational noise as an important risk factor for hearing loss. We found that men, nonHispanic white and the age group of $40-59$ years were more susceptible to occupational noise-associated hearing loss than other groups.

In fact, we ran regression analyses dealing with O*NET scores as a continuous variable and estimated the $\beta$ coefficients corresponding to a one-unit increase in O*NET scores. The OR for risk of hearing loss corresponding to a one-unit increase in O*NET scores (range between 1 and 5) was 1.65 (95\% CI 1.28 to 2.13 ) in a multivariable-adjusted model. A significant dosedependent relationship with $\mathrm{O}^{*} \mathrm{NET}$ scores was retained in sequential models after adjusting for socioeconomic factors, non-occupational noise exposures and other potential risk factors. This suggests that the association between occupational noise exposure and hearing loss is independent of such risk factors. This increased risk is roughly equivalent to 20 or more pack-years of smoking $(\mathrm{OR}=1.54)$, diabetes $(\mathrm{OR}=1.66)$ and recreational noise exposure $(\mathrm{OR}=1.62)$ (see supplemental table 8). The estimated effect size of O*NET score is also similar to the effects of 5 years of ageing $(O R=1.69)$ when age is fit linearly.

It is difficult to compare our findings to other studies because there are no studies of dose-response relationship between occupational noise exposure and hearing loss in the general population with low to high exposure as a continuous variable. A few previous investigations of noise and hearing loss have been made across crude occupational groups in the general population. In one such study, over 3500 older adults in Beaver Dam, Wisconsin, were examined for hearing loss in six occupation categories. A statistically significant increased risk of hearing loss was found in service (OR=1.85, 95\% CI 1.40 to 2.43), operations/fabricators ( $\mathrm{OR}=1.99,95 \%$ CI 1.53 to 2.59) and production $(\mathrm{OR}=3.48,95 \% \mathrm{CI} 2.53$ to 4.79$)$ compared to management as a reference group. ${ }^{6}$ Another study mailed a questionnaire over 22000 adults of working age across Britain and examined the association between years worked in a noisy job and self-reported hearing difficulty. That study found an increase in hearing difficulty by years worked in a noisy job and a statistically significant increased risk of hearing loss in $5-10$ years (prevalence ratio $=3.0,95 \%$ CI 1.5 to 6.1 ) and over 10 years (prevalence ratio $=3.8,95 \%$ CI, 2.4 to 6.2 ) compared to non-exposed group. ${ }^{16}$ Our findings are broadly compatible with these studies and confirm the evidence of increased risk of hearing loss with increase in occupational noise exposure in the general population.
In addition to its relationship with hearing loss, the O*NET occupational noise score had a strong dose-dependent relationship with noise notch. The presence of noise notch is one diagnostic in determining that hearing loss is noise induced rather than the effect by other factors such as ageing. ${ }^{11}{ }^{12}$ The ORs of the risk of noise notch increased also gradually across the O*NET noise score quintiles (ORs 1 (Reference), 0.96, 0.79, 1.35 and 1.51). This suggests that the $\mathrm{O}^{*} \mathrm{NET}$ occupational noise could be a good proxy for occupational noise exposure.

The main strengths of this study include (1) the use of representative samples of the US general population, including over sampled minority populations, which enables the observed results to be generalisable; (2) the adjustment for various potential confounding factors of the association between occupational noise and hearing loss, especially noise exposure other than workplace noise, such as firearm and recreational noise, and use of ototoxic medication and (3) the use of NHANES data conducted with strict quality control procedures.

This study has several limitations that should be considered. Because the O*NET database we used is based on the frequency of exposure to sounds and noise levels considered distracting and uncomfortable rather than on actual noise measurements, exposure misclassification may exist. Moreover, the O*NET data are classified only by occupation groups and do not account for variations in noise exposure from different industry groups or different job task groups within the same occupation classification. The assumption that jobs with the same title have similar occupational noise exposure could also lead to misclassification of exposure. Misclassification might also have occurred when $801 \mathrm{O}^{*} \mathrm{NET}$ occupation groups were combined into 40 NHANES occupation groups. Because the O*NET survey is totally independent of the audiometry tests in NHANES, however, such exposure misclassification is likely to be non-differential and leads to a true association towards the null.

Although our study showed that as an exposure proxy, longest job is better than current job in predicting occupational noise-induced hearing loss, we could not account for the job history nor the duration of each job. Because the reported longest job is more likely to be related to hearing loss, however, the bias would be non-differential. Collecting information on full job history and duration would improve validity and reliability of any noise exposure assessment using O*NET.

Although we examined three cycles of the NHANES data, which offers significant power, causal inferences may not be made because of the cross-sectional nature of the NHANES data. Nevertheless, use of the longest job may be temporally relevant to current audiometry test results.

One might argue that there is selection bias in that the association between occupational noise and hearing loss is different for subjects included in our analysis who provided information on their longest job and those excluded due to no longest job information. We found that the prevalence of noise notch for included subjects was significantly different from the prevalence for excluded subjects and that included subjects were more likely than excluded subjects to have been exposed to loud job noise for at least 3 months on all previous jobs (supplemental table 2). Most of the excluded subjects had never worked $(75 \%)$, are currently housewives (67\%, all women), disabled people with no job history $(10 \%)$ and students (8\%). Although our results cannot be generalised to the non-included people (housewives, students and the disabled), we believe that the observed associations are valid to conclude noise exposure at workplaces as an important risk factor for hearing loss and that the selection bias is unlikely. 
In summary, the present study supports the hypothesis that occupational noise exposure increases the risk of hearing loss across various occupations. Utilisation of the O*NET noise exposure data would allow us to perform epidemiological studies of occupational noise exposure in the general population and to better understand the health effects of occupational noise exposure.

Funding This study was supported by the National Institute of Environment Health Sciences grants K01-ES016587. This research was also supported in part by a pilot project research training grant from the Center for Occupational Health and Safety Engineering (COHSE) at the University of Michigan. COHSE, an Education and Research Center, is supported by training grant No. 2T42OH008455 from the Centers for Disease Control and Prevention/National Institute for Occupational Safety and Health. The contents of this paper are solely the responsibility of the author(s) and do not represent official views of the National Institute for Occupational Safety and Health. Other funders: National Institutes of Health.

Competing interests None declared.

Patient consent Obtained.

Provenance and peer review Not commissioned; externally peer reviewed.

\section{REFERENCES}

1. Bainbridge KE, Hoffman HJ, Cowie CC. Diabetes and hearing impairment in the United States: audiometric evidence from the National Health and Nutrition Examination Survey, 1999 to 2004. Ann Intern Med 2008;149:1-10.

2. Pleis JR, Lethbridge-Cejku M. Summary health statistics for U.S. adults: National Health Interview Survey, 2005. Vital Health Stat 10 2006;(232):1-153.

3. Stanbury M, Rafferty AP, Rosenman K. Prevalence of hearing loss and work-related noise-induced hearing loss in Michigan. J Occup Environ Med 2008;50:72-9

4. Burr H, Lund SP, Sperling BB, et al. Smoking and height as risk factors for prevalence and 5 -year incidence of hearing loss. A questionnaire-based follow-up study of employees in Denmark aged 18-59 years exposed and unexposed to noise. Int J Audiol 2005;44:531-9.

5. Rubak T, Kock SA, Koefoed-Nielsen B, et al. The risk of noise-induced hearing loss in the Danish workforce. Noise Health 2006;8:80-7.

6. Cruickshanks KJ, Wiley TL, Tweed TS, et al. Prevalence of hearing loss in older adults in Beaver Dam, Wisconsin: the Epidemiology of Hearing Loss Study. Am J Epidemiol 1998;148:879-86.

7. Tak S, Calvert GM. Hearing difficulty attributable to employment by industry and occupation: an analysis of the National Health Interview Survey-United States, 1997 to 2003. J Occup Environ Med 2008;50:46-56.

8. Nelson DI, Nelson YN, Concha-Barrientos M, et al. The global burden of occupational noise-induced hearing loss. Am J Ind Med 2005;48:446-58.

9. Tak S, Davis RR, Calvert GM. Exposure to hazardous workplace noise and use of hearing protection devices among US workers-NHANES, 1999-2004. Am J Ind Med 2009;52:358-71.

10. Hearing Loss Prevention: Occupational Safety and Health Risks National Institute for Occupational Safety and Health. http://www.cdc.gov/niosh/programs/hlp/risks.html (accessed May 2010).
11. Coles RR, Lutman ME, Buffin JT. Guidelines on the diagnosis of noise-induced hearing loss for medicolegal purposes. Clin Otolaryngol Allied Sci 2000:25:264-73

12. Rabinowitz PM, Galusha D, Slade MD, et al. Audiogram notches in noise-exposed workers. Ear Hear 2006;27:742-50.

13. Beckett WS. Noise induced hearing loss-evidence based statement. J Occup Environ Med 2003:45:1029.

14. Mannetje A, Kromhout $\mathrm{H}$. The use of occupation and industry classifications in general population studies. Int J Epidemiol 2003;32:419-28.

15. Teschke K. Exposure surrogates: job-exposure matrices, self-reports, and expert evaluations. In: Nieuwenhuijsen MJ, ed. Exposure Assessment in Occupational and Environmental Epidemiology. New York, NY: Oxford University Press, 2003:119-32.

16. Palmer KT, Griffin MJ, Syddall HE, et al. Occupational exposure to noise and the attributable burden of hearing difficulties in Great Britain. Occup Environ Med 2002;59:634-9.

17. Cifuentes M, Boyer J, Lombardi DA, et al. Use of O*NET as a job exposure matrix: a literature review. Am J Ind Med 2010;53:898-914.

18. Alterman T, Grosch J, Chen X, et al. Examining associations between job characteristics and health: linking data from the Occupational Information Network (0*NET) to the US national health surveys. J Occup Environ Med 2008; 50:1401-13.

19. O*NHET Resource Center. The O*NET® Content Model. US Department of Labor http://www.onetcenter.org/content.html (accessed Jan 2008).

20. National Crosswalk Service Center, O*NET Occupations Reference Guide. Des Moines, IA, 2007.

21. 0*NET Resource Center. Generic Questionnaires: O*NET Work Context Questionnaire. US Department of Labor. http://www.onetcenter.org/questionnaires. html (accessed Jan 2008)

22. National Health and Nutrition Examination Survey. NHANES 1999-2000 Data Documentation: Audiometry. Hyattsville, MD: National Center for Health Statistics, The Centers for Disease Control and Prevention, 2005.

23. Gates GA, Mills JH. Presbycusis. Lancet 2005;366:1111-20.

24. Agrawal Y, Platz EA, Niparko JK. Prevalence of hearing loss and differences by demographic characteristics among US adults: data from the National Health and Nutrition Examination Survey, 1999-2004. Arch Intern Med 2008; 168:1522-30.

25. Ikeda N, Murray CJ, Salomon JA. Tracking population health based on self-reported impairments: trends in the prevalence of hearing loss in US adults, 1976-2006. Am J Epidemiol 2009;170:80-7.

26. National Health and Nutrition Examination Survey. NHANES 1999-2000 Data Documentation. Hyattsville, MD: National Center for Health Statistics, 2008

27. National Health and Nutrition Examination Survey. Codebook for Data Production (1999-2000). Hyattsville, MD: National Center for Health Statistics, 2006.

28. View Census 2000 Classified Index of Occupations Mapped to SOC- Sorted by SOC Code. U.S. Census Bureau, Housing and Household Economic Statistics Division, 2009. http://www.census.gov/hhes/www/ioindex/view.html (accessed Jan 2008).

29. National Health and Nutrition Examination Survey. NHANES Audiometry Procedures Manual. Hyattsville, MD: National Center for Health Statistics, The Centers for Disease Control and Prevention, 2005

30. Agrawal Y, Platz EA, Niparko JK. Risk factors for hearing loss in US adults: data from the National Health and Nutrition Examination Survey, 1999 to 2002. Otol Neurotol 2009;30:139-45

Visit OEM online for free editor's choice articles, top ten articles, online archive, blogs and online first articles. Keep informed and up to date by registering for electronic table of contents at oem. bmj.com. 